\title{
Driver Assistance System for Lane Detection and Vehicle Recognition with Night Vision
}

\author{
Chun-Che Wang', Shih-Shinh Huang', and Li-Chen $\mathrm{Fu}^{1,2}$ \\ Department of Computer Science and Information \\ Engineering ${ }^{l}$, \\ Department of Electrical Engineering ${ }^{2}$, \\ National Taiwan University, Taipei, Taiwan, R.O.C.
}

\begin{abstract}
The objective of this research is to develop a vision-based driver assistance system to enhance the driver's safety in the nighttime. The proposed system performs both lane detection and vehicle recognition. In lane detection, three features including lane markers, brightness, slenderness and proximity are applied to detect the positions of lane markers in the image. On the other hand, vehicle recognition is achieved by using an evident feature which are extracted through three four steps: taillight standing-out process, adaptive thresholding, centroid detection, and taillight pairing algorithm. Besides, an automatic method is also provided to calculate the tilt and the pan of the camera by using the position of vanishing point which is detected in the image by applying Canny edge detection, Hough transform, major straight line extraction and vanishing point estimation. Experimental results for thousands of images are provided to demonstrate the effectiveness of the proposed approach in the nighttime. The lane detection rate is nearly $99 \%$, and the vehicle recognition rate is about $91 \%$. Furthermore, our system can process the image in almost real time.
\end{abstract}

Index Terms - Night Vision, Lane Detection, Vehicle Recognition, Vanishing Point Detection, Driver Assistance System

\section{INTRODUCTION}

In Taiwan, more than 2,500 people die in the fatal traffic accidents per year, of which $53 \%$ traffic accidents happen in the nighttime. The major cause of traffic accidents is "Improper Driving" due to driver's inattention and fatigue. The researches on Driver Assistance System (DAS) have been conducted generally in Intelligent Transportation System (ITS) for many years. For developing a DAS at night, some researches often select vision-based sensing device that could provide abundant environment information.

\section{A. Related Works}

According to electromagnetic spectrum, nowadays visionbased researches can be categorized into three classes according to the range of operating wavelength, namely, visible spectrum region $(0.38 \sim 0.8 \mu \mathrm{m})$, Near Infra-Red region (NIR) $(0.8 \sim 1.2 \mu \mathrm{m})$, and Far Infra-Red region (FIR) $(7 \sim 14 \mu \mathrm{m})$. However, human cannot perceive the wavelength of the above region expect the visible spectrum, and they can see the NIR or FIR images through the sensors which are capable of detecting energy radiated by a band of the electromagnetic spectrum.

Among the devices of night vision, the FIR device can detect the presence of objects that emit heat, such as vehicles [1], pedestrians [2], and animals, etc. The advantage of such

\author{
Pei-Yung $\mathrm{Hsiao}^{3}$ \\ Department of Electronic Engineering ${ }^{3}$,
}

Chang Gung University, Tao-Yuan, Taiwan, R.O.C.

devices is that they work well under various illumination conditions even in the nighttime. But, they are sensitive to different kinds of weather, such as rain and snow. Besides, these devices are usually expensive.

The NIR device can detect the objects regardless of the light situation even under dark condition. In the NIR image based research, Pavlidis et al. [3] proposed a fuzzy neural network classifier to operate upon the fused NIR image for automated counting of vehicle occupants day and night. Jahard et al. [4] combined the benefit of the NIR and of FIR images to improve the automotive night vision device. Besides, we had tested the night images by using NIR devices with highlighted CCD camera and near-infrared illuminator. However, the testing results show that this kind of IR device is not suitable for the night images.

For cost and performance considerations, the CCD camera which operates in the visible spectrum band is taken as our sensing device to develop a night DAS system. There are a few researches that are based on the visible spectrum images to develop a night DAS. In [5], a deformable template method is used to optimize a likelihood function based on the proposed model. However, the simulated annealing optimization algorithm cannot guarantee a global optimum solution and consume huge computational resources.

Another research work [6] models the lane boundaries as a curve in the form of a second-order polynomial by using image objects corresponding to reflected spots on the ground plane. However, the reflected spots sometimes are broken in some sections of a highway and are occluded by the frontvehicles in the image. So, it is difficult to recover the lane boundaries from the limited number of reflected spots in the images.

In [7], they assume that the headlight is the bright blobs relative to the dark background. Next, they perform binarization to extract the headlights of the oncoming vehicles on the highway at night. However, the drawback of this approach is that the detected results are easily affected by the other bright blobs, such as streetlamps, reflected spots, and the beams of headlights.

In [6], the rear-light is assumed to be in the form of a white bright region surrounded by red pixels. However, the red and white pixels will be falsely detected by a fixed threshold under different illumination. Besides, the above assumption made for rear-light may be incorrect because the shapes of them are different in braking and moving situations. B. Organization 
This paper is organized as follows. In section II, the camera configuration and camera calibration based on vanishing point detection is first introduced. The lane boundary detection procedure is developed in section III, and the vehicle recognition procedure at night is presented in section IV. Finally, the experimental results and conclusion are described in section V and section VI, respectively.

\section{CAMERA CALIBRATION}

\section{A. Overview}

Camera calibration is a mean to determine the parameters of transformation between the image coordinate and the world coordinate. The most typical approaches with monocular camera, such as DLT method [8], are to model the transformation as parametric linear transformation. Thus, the parameters are solved based on a set of known corresponding points in the world coordinate system and the ones in the image coordinate system. However, it is inconvenient to physically measure a larger quantity of points in the world coordinate system.

Furthermore, another research [9] is to measure the height and the tilt of the camera. Based on the information of the given vanishing point, the focal length and the pan of the camera can be computed. But the tilt of camera is still hard to obtain without any measuring instruments.

In this paper the proposed method does not require any measuring instruments. The main idea is to compute the tilt and the pan angles of the camera according to the image position of vanishing point, which is detected by the use of image processing technique.

\section{B. Camera Configuration}

We define two coordinates systems, namely, vehicle coordinate system and image coordinate system. The origin of the vehicle coordinate system is on the flat ground plane which is right under the camera, as shown in 0 . The $Y$-axis is parallel with the driving direction which is controlled by the steering wheel. On the other hand, the image coordinate system is the image plane which is in perpendicular to camera optical axis. $U$-axis is in horizontal direction and $V$-axis is in vertical direction. We take the top-leftmost position of the image plane as the origin.

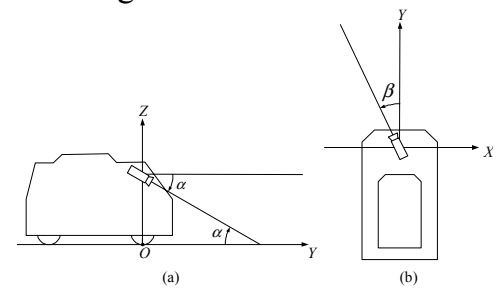

Fig. 1 Vehicle coordinate system. (a)Side view. (b)Bird's eye view.

In general, tilt angle $\alpha$ and pan angle $\beta$ of the camera are taken into account for describing the camera configuration (See 0 ). In addition, the pinhole model is applied in the system such that the transformation formulas from image coordinate $(u, v)$ to vehicle coordinate $(x, y)$ could be derived as follows:

$$
y=\frac{K_{v} \cdot h-(v-H / 2) \cdot h \tan \alpha}{(v-H / 2)+K_{v} \cdot \tan \alpha},
$$

$$
x=\frac{y \cdot(u-W / 2)-K_{u} \cdot y \tan \beta}{K_{u}+(u-W / 2) \cdot \tan \beta},
$$

In (1) and (2), $h$ is the camera height from the ground plane, $f$ is the focal length of the camera. $H, W$ are the height and width of the image, respectively. $K_{v}, K_{u}$ are the camera constants in the vertical and horizontal directions. Furthermore, $K_{v}=f / \Delta v, K_{u}=f / \Delta u$ where $\Delta v, \Delta u$ are the scaling factors of the image plane in the vertical and horizontal directions, respectively.

Because the distance between vanishing point and host vehicle is infinite, by letting $y$ tend to infinity, we have:

$$
\tan \alpha=\left(\frac{H}{2}-v_{v}\right) / K_{v}, \tan \beta=\left(u_{v}-\frac{W}{2}\right) / K_{u},
$$

where $\left(u_{v}, v_{v}\right)$ denotes the image coordinate of the vanishing point. Two unknown parameters, $\alpha$ and $\beta$, can be computed straightforward by (3).

\section{Vanishing Point Detection}

Vanishing point is a point in the image plane, to which a set of parallel lines in the 3D space will converge. Hence, in the first place, we perform Canny edge detection [10] to extract the edge pixels in the image, and perform connected component labeling with 8-neightborhood [11] to remove the details. Finally, the Canny edge map is shown in Fig. 2(b).

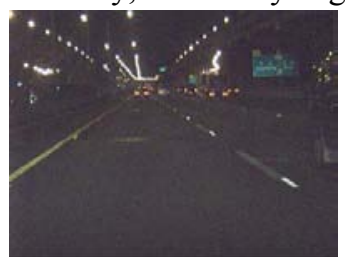

(a)

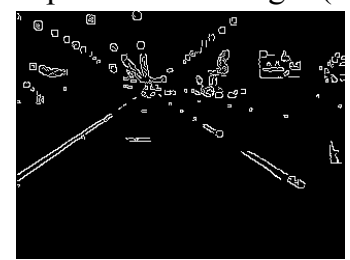

(b)
Fig. 2 (a )Original image. (b)Canny edge map.

In order to detect the all the straight lines in the image, Hough Transform [11] is executed with voting scheme in the polar parameter space $(d, \theta)$. For a straight line, $L$, it can be expressed as $d=u \cdot \cos \theta+v \cdot \sin \theta$ in the image coordinate system, where $d$ is the perpendicular distance from $L$ to the origin of the image; $\theta$ is the included angle which is calculated clockwise from the $U$-axis whichever is perpendicular to $L$ and passes through the origin of the image.

Here, $\theta$ is restricted in the range $[0,2 \pi]$ whereas $d$ lies in the range $\left[0, \sqrt{H^{2}+W^{2}}\right]$. In our experiment, $\sqrt{H^{2}+W^{2}}$ is equal to 400. Afterwards, for every detected edge pixel, we give a vote to all possible parameters that corresponds to a line passing through it. 0(a) shows the result of Hough Transform in parameter space, the brighter point in the figure corresponds to the parameter that has higher votes.

In our application, we assume that the camera is installed without any orientation respect to the ground plane. Under this assumption, the vertical and the horizontal straight lines could be neglected because both of them rarely pass through the vanishing point. 0 (b) shows the result after discarding the vertical and horizontal straight lines. 


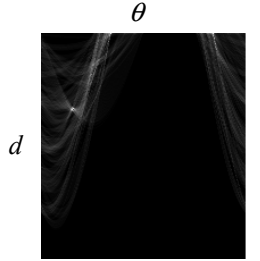

(a)

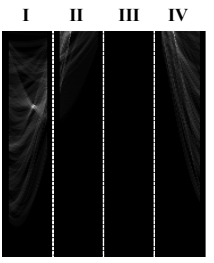

(b)

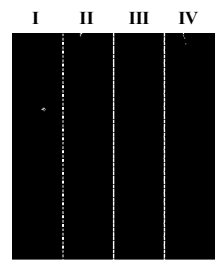

(c)
Fig. 3 Polar parameter space.

Furthermore, we take several points in the parameter space with high confidence as the major straight lines for detecting the vanishing point. According to $\theta$-values, the straight lines in the image can be divided into four quadrants, I: $[0, \pi / 2)$, II: $[\pi / 2, \pi)$, III: $[\pi, 3 \pi / 2)$ and IV: $[3 \pi / 2,2 \pi)$. The local thresholding strategy in each quadrant is then applied.

The reason for using local thresholding strategy is that it takes the lines in different directions into account and hence gets better result than using the global one. Therefore, we define a certain percentage of local maximum votes as a threshold in each quadrant. 0 (c) shows the major straight lines in the polar parameter space after local thresholding strategy. And 0(a) shows the major straight lines in the image.

As mentioned before, vanishing point is nearly intersected by all the major straight lines. But, the major straight lines with higher confidence should be more significant. For this reason, the vanishing point is a point that minimizes the weighted summation of distance from all major straight lines to it [12]. And the least square method is applied to reduce the estimation error. Finally, the result of vanishing point detection is shown in $0(\mathrm{~b})$ and the white ' + ' denotes the position of the vanishing point.

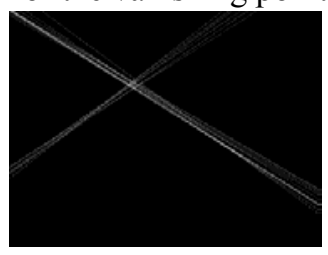

(a)

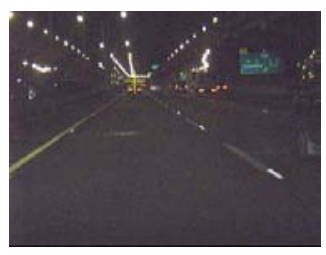

(b)
Fig. 4 (a)Major straight lines. (b)Vanishing point detection.

\section{LANE DETECTION}

In our system, the objective of lane detection is to locate the lane boundaries with the painted lane markers under various conditions. By close observation, we can find out three properties of painted lane markers, they are brightness, slenderness and proximity [13].

\section{A. Feature Point Extraction}

According to the brightness and slenderness properties, Peak-Finding Algorithm [13] is used to extract the feature points of lane markers in the image. However, the fixed threshold in the image is not adapted to different illumination at night. For this reason, we propose an automatic mechanism to adjust the threshold according to the surrounding illumination.

An adaptive threshold $t^{*}$ as will be defined below:

$$
t^{*}=\arg \min _{t}\left\{|S(t)|>\gamma \cdot\left|U_{\text {Hill }}\right|\right\} \text {, }
$$

where $U_{\text {Hill }}$ denotes the global set of the peaks detected in the image; $S(t)$ is a subset of $U_{\text {Hill }}$ containing the peaks whose height are smaller than $t ;\left|U_{\text {Hill }}\right|$ and $|S(t)|$ are the numbers of peaks in $U_{\text {Hill }}$ and $S(t)$, respectively; $\gamma$ is the ratio number in the range $[0,1]$. If the detected peak which height is small than $t^{*}$, we will discard it.

Because the lane markers are located on the ground plane, we can only focus on the region below the vanishing line. This idea is shown in Fig. 5(b).

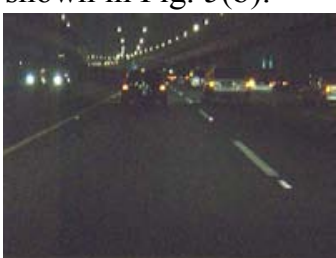

(a)

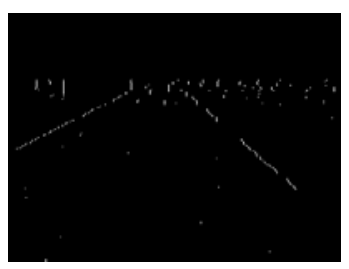

(b)
Fig. 5 (a)Original Image. (b)Results of feature point extraction.

\section{B. Feature Points Grouping}

For easy implementation, we convolute the image by use of a $3 \times 9$ neighboring mask as shown in Fig. 6(a). The mask is designed for covering all possible lane markers with different slopes in the image. Therefore, the neighboring feature points will belong to the same line segment after the convolution operation.

However, noise points will make the direction of line segments diverge. In order to represent a line segments in a more precise way, a least square method is applied to characterize the line segment as shown in Fig. 6(b).

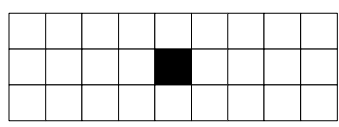

(a)

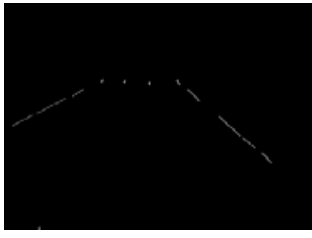

(b)
Fig. 6 (a) Neighboring mask. (b)Line segment image.

\section{Line Segment Combination}

According to proximity property, line segments belonging to the same lane boundary have two specific characteristics present in the image. First, the slopes of them are the same. Second, for two line segments, either top or bottom point of one line segment will locate precisely on the extended line of the other.

For error tolerance, two quantitative measurements, $\rho_{i j}$ and $\phi_{i j}$, are introduced to evaluate these two characteristics. Given two line segments, $L_{i}$ and $L_{j}, \rho_{i j}$ is the minimum perpendicular distance between $L_{i}$ and $L_{j}$. On the other hand, $\phi_{i j}$ is an included angle between $L_{i}$ and $L_{j}$ (See Fig. 7(c)). Besides, $\rho_{i}$ is the perpendicular distance from $L_{j}$ to the bottom feature point of $L_{i} ; \rho_{j}$ is the perpendicular distance from $L_{i}$ to the top feature point of $L_{j}$. Fig. 7(a)(b) show these cases.

Two measurements for characteristic evaluation.

Therefore, $\rho_{i j}$ is defined as follows:

$$
\rho_{i j}=\min \left\{\rho_{i}, \rho_{j}\right\},
$$

in addition, $\phi_{i j}$ is computed by the following equation:

$$
\phi_{i j}=\cos ^{-1}\left(\left|\frac{1+m_{i} \cdot m_{j}}{\sqrt{\left(1+m_{i}^{2}\right) \cdot\left(1+m_{j}^{2}\right)}}\right|\right) \text {, }
$$


where $m_{i}, m_{j}$ are the slopes of $L_{i}$ and $L_{j}$, respectively.

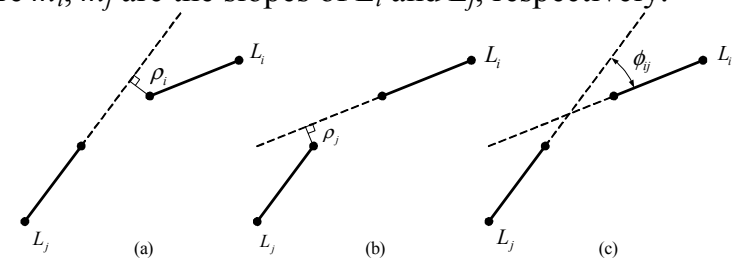

Fig. 7 Two measurements for characteristic evaluation.

Afterwards, the confidence function is introduced to determine whether $L_{i}$ and $L_{j}$ belong to the same lane boundary according to $\rho_{i j}$ and $\phi_{i j}$. The confidence function is defined as:

$$
\text { Conf }_{\text {combination }}\left(\rho_{i j}, \phi_{i j}\right)=f\left(\rho_{i j}\right) \cdot f\left(\phi_{i j}\right) \text {, }
$$

where $f(x)=1 /\left(1+\omega_{x} \cdot x^{2}\right)$ is the function of criterion $x$, and $\omega_{x}$ determines how fast $f(x)$ decreases with $x$. It is interesting to see that, $f(x)$ always falls in the range $[0,1]$. Finally, the line segments are combined to form several lane boundary candidates as shown in Fig. 8(a).

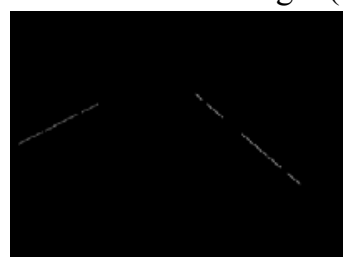

(a)

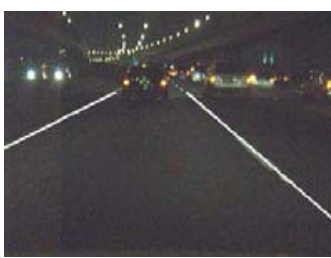

(b)
Fig. 8 (a)Lane boundary candidates. (b)The result of lane detection.

\section{Lane Boundary Selection}

Sometimes false results will be detected by the proposed method. The most probable candidates of the lane boundaries are selected by the verification of their vehicle coordinates. The result of lane detection is shown in Fig. 8 (b).

\section{VEHICLE RECOGNITION}

By our observation, the taillight is the only feature of preceding vehicle at night. Besides, on highway, the vehicles driven in the opposite directions are often divided by the traffic islands and such situation rarely causes traffic hazards. Hence, we can discard the vehicle driven in the opposite direction; on the contrary, the preceding vehicles often bring the potential hazards for the host vehicle.

\section{A. Taillight Extraction}

According to the analysis of the taillight in $\mathrm{R}$ and $\mathrm{B}$ color channels, the $r$ value of the pixels in the taillight region is extremely high, and the difference between $r$ value and $b$ value of these pixels is usually quite large. In addition, the $r$ value of the pixels in the headlight region is nearly equal to its $b$ value. Under this observation, we stand out the taillight pixels and suppress headlight pixels by subtracting $b$ value from $r$ value directly.

Let $I_{R-B}(u, v)=R(u, v)-B(u, v)$ be the result image after subtraction, where $R(u, v)$ and $B(u, v)$ denote the $r$ value and $b$ value of the pixel position $(u, v)$, respectively. Then, we translate the $I_{R-B}$ to the image, $N_{R-B}$, by normalizing the value of each pixel in the $I_{R-B}$ image to the range $[0,255]$. To remove noise effect duo to exposure time and environmental illumination, we adopt Gaussian filter as our noise removing operator.
Therefore, $N_{R-B}$ after of noise removal is shown in Fig. 9(a). We can see that the intensity values of the taillight are relatively higher than other objects. On the contrary, in the left part of Fig. 9(a), the intensity values of headlights will be suppressed, which in turn discards the vehicles in the opposite driving direction. In order to overcome different illumination at night, an adaptive threshold according to the histogram of $I_{R-B}$ is applied to extract taillight pixels (See Fig. 9).

Although the shapes of taillights after taillight extraction are varied depending on the vehicle (see Fig. 10), the shapes of taillights is near circular no matter how the vehicle is moving or braking even through it is compound.

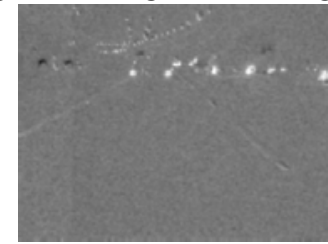

(a)

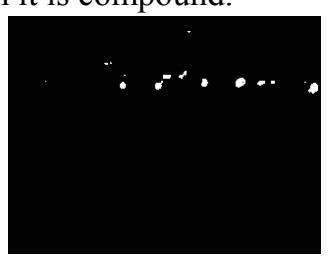

(b)
Fig. 9 (a)Taillights standing out. (b)Taillight extraction.

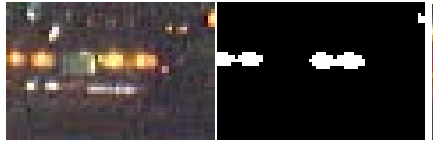

(a) Compound taillight

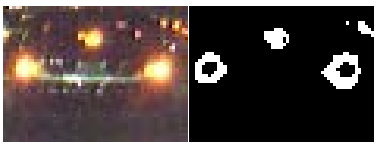

(b) Taillights in braking in state
Fig. 10 The shapes of taillights in different states

\section{B. Taillight Pairing Algorithm}

On the highway, the typical vehicles are passenger car, recreation vehicle, jeep, and small freight car. Furthermore, the vehicles are more or less about 170 centimeters wide. Furthermore, from driver's view, the aspect ratio (width/height) of vehicle is usually approximate to 2.0.

Therefore, the proposed taillight pairing algorithm is according to the above constraints. However, the other unusual vehicles such as big freight car, truck, and big bus do not satisfy the above constraints and so they are not considered in our research work. For every two taillight centroids, $C_{i}\left(u_{i}, v_{i}\right)$ and $C_{j}\left(u_{j}, v_{j}\right)$, taillight paring algorithm is as follows:

Step 1: $\left|v_{i}-v_{j}\right|>\Delta h$, go to Step 6, where $\Delta h$ is a tolerant value caused by centroid detection process.

Step 2: Let $V C\left(C_{i}, C_{j}\right)$ be the vehicle candidate containing $C_{i}$ and $C_{j}$. The width of $V C$ is defined as $\left|u_{i}-u_{j}\right|$, and the height of $V C$ is a half of the width.

Step 3: The bottommost $v$-values of $V C$, called $v_{\text {bottom }}$, is defined as $\min \left\{v_{i}, v_{j}\right\}+\left|u_{i}-u_{j}\right| 2$. If $v_{\text {bottom }}$ is out of detection range, go to Step 6.

Step 4: If the width of $V C$ is greater than $180 \mathrm{~cm}$ or is less than $160 \mathrm{~cm}$, go to Step 6 .

Step 5: $V C$ is likely a vehicle candidate, and return true.

Step 6: $C_{i}$ and $C_{j}$ can not form a vehicle candidate, and return false.

Sometimes, the vehicle candidates are suddenly missing for a while in the image, because the host vehicle vibrates up or down slightly on the rugged highway section. Consequently, the vibration error should be taken into consideration so that the width constraint for pairing is relaxed 
somewhat. Finally, the result of taillight paring algorithm is shown in Fig. 11(a), and the preceding vehicle could be filtered by detected lane boundaries (See Fig. 11(b)).

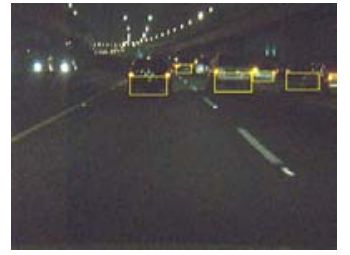

(a)

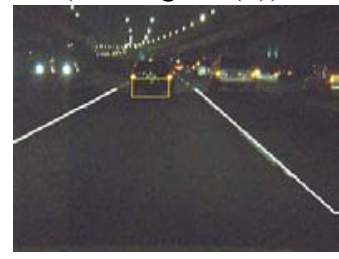

(b)
Fig. 11 (a)Vehicle candidates. (b)Preceding vehicle recognition.

\section{EXPERIMENT}

\section{A. Experiment Description}

A CCD camera is mounted behind the driving mirror on the experimental passenger car (See Fig. 12), and it is used to continuously monitor the traffic scenes at the driving speed between $30 \mathrm{~km} / \mathrm{hr}$ and $100 \mathrm{~km} / \mathrm{hr}$. There already exist some mechanisms which can achieve stability of the vehicle and the camera nowadays, so that the vibration problem can be ignored in our experiment. The grabbed images $(320 \times 240)$ are delivered to the mobile computer which is equipped with Intel Pentium IV 1.8GHz processor and 320MB RAM through IEEE 1394 interface.

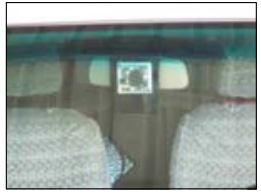

(a) Front view

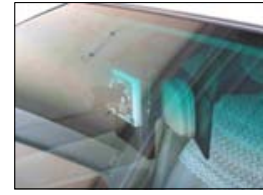

(b) Side view

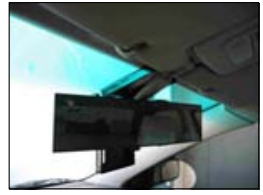

(c) Back view
Fig. 12 Environmental setup

\section{B. Detection Result}

Sometimes, the results of lane detection are affected by the reflected spots on the ground, the vibration of vehicle, and the taillights. These failed situations are respectively shown in Fig. 13(a)(b)(c). However, these temporal situations could be recovered by lane tracking according to the previous frames.

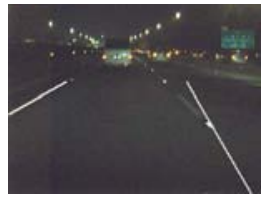

(a) Affected by reflected spots

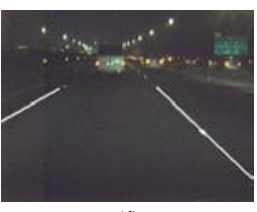

(d)

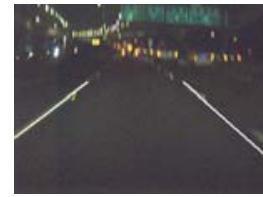

(b) Vehicle vibration

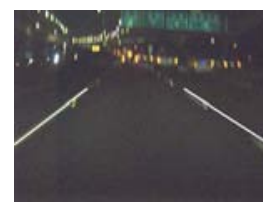

(e)

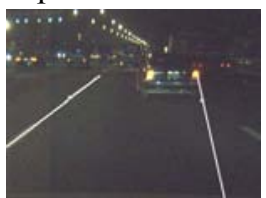

(c) Affected by taillight

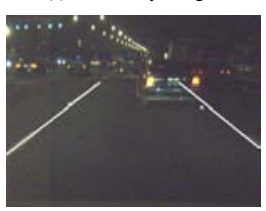

(f)
Fig. $13(a)(b)(c)$ are the failed situations of lane detection. (d)(e)(f) are the corresponding images which are recovered by lane tracking.

1230 real traffic night images are used to test our system which both vehicle recognition and lane detection are integrated. The failed situations include false detection and miss. In lane detection process, the detection rate is $98.53 \%$; on the other hand, the detection rate in vehicle recognition within 40 meters is $90.51 \%$. Error! Reference source not found. shows the experimental results of our system.

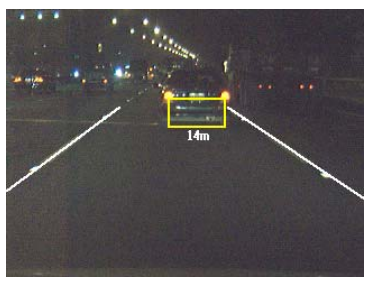

(a)

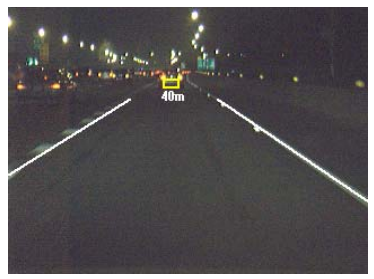

(c)

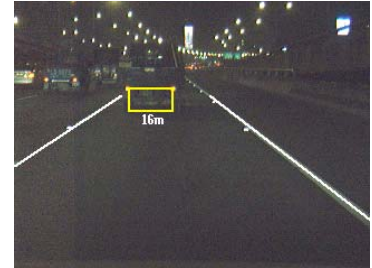

(b)

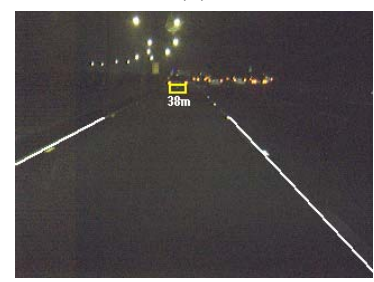

(d)
Fig. 14 Detection results.

\section{C.System Performance}

To facilitate the analysis more clearly, each procedure in our system is decomposed into several modules. Then, we compute the average processing time in each module according to the whole images. In addition, the accuracy of the processing time is reached at the microsecond level $\left(10^{-6}\right.$ second). Table I shows the processing time of the vanishing point detection algorithm. Table II shows the processing time of the lane detection and the vehicle recognition procedures. TABLE I

PROCESSING TIME OF VANISHING POINT DETECTION PROCEDURE

\begin{tabular}{|c|c|c|c|}
\hline \multirow{2}{*}{$\begin{array}{c}\text { Vanishing } \\
\text { Point } \\
\text { Detection }\end{array}$} & Canny Edge detection & $57.5 \mathrm{~ms}$ & \multirow{2}{*}{$158.2 \mathrm{~ms}$} \\
\cline { 2 - 3 } & Hough Transform & $90.09 \mathrm{~ms}$ & \\
\cline { 2 - 3 } & Others & $10.61 \mathrm{~ms}$ & \\
\hline
\end{tabular}

Processing TIME OF SySTEM

\begin{tabular}{|c|c|c|c|}
\hline \multirow{5}{*}{ Lane Detection } & Gaussian Filter & $10.49 \mathrm{~ms}$ & \multirow{5}{*}{$17.48 \mathrm{~ms}$} \\
\hline & Feature Point Extraction & $2.36 \mathrm{~ms}$ & \\
\hline & Feature Point Grouping & $1.84 \mathrm{~ms}$ & \\
\hline & Line Segment Combination & $1.53 \mathrm{~ms}$ & \\
\hline & Lane Boundary Selection & $1.49 \mathrm{~ms}$ & \\
\hline \multirow{5}{*}{$\begin{array}{c}\text { Vehicle } \\
\text { Recognition }\end{array}$} & Taillight Pixel Standing-Out & $7.43 \mathrm{~ms}$ & \multirow{5}{*}{$22.93 \mathrm{~ms}$} \\
\hline & Gaussian Filter & $10.61 \mathrm{~ms}$ & \\
\hline & Taillight Pixel Filtering & $0.92 \mathrm{~ms}$ & \\
\hline & Taillight Centroid Detection & $2.76 \mathrm{~ms}$ & \\
\hline & Taillight Pairing Algorithm & $0.88 \mathrm{~ms}$ & \\
\hline
\end{tabular}

Therefore, the system is effective and only takes $41 \mathrm{~ms}$ to detect the lane boundary and the preceding vehicle at the same time. As we can see, this overall procedure can run in near real-time.

\section{CONCLUSION}

In this paper, we provide a night-vision based driver assistance system on highway environment to enhance the driver's safety. Furthermore, we also give an automatic mechanism to compute the parameters of camera without measuring instruments. Finally, the experimental results demonstrate the effectiveness and efficiency of our proposed system.

For lane detection, the Peak-Finding Algorithm is proposed to extract the feature points effectively based on the 
lane markers' characteristics. Then, the lane markers are detected by grouping the feature points. Next, the line segments are combined into a lane boundary by confidence function. Finally, the lane boundaries of the main lane are selected in the image successfully. On the other hand, vehicle recognition is achieved according to the effective extraction of taillights. Afterwards, the vehicle candidates are localized successfully according to taillight pairing algorithm.

In the near future, we will integrate a warning mechanism based on time-to-collision (TTC) criteria and the detection results to warn the driver at the proper time.

\section{REFERENCES}

[1] T. Tsuji, H. Hattori, M. Watanabe, and N. Nagaoka, "Development of Night-Vision System," IEEE Transactions on Intelligent Transportation Systems, Vol. 3, No. 3, pp. 203-209, Sept. 2002.

[2] L. Andreone, P. C. Antonello, M. Bertozzi, A. Broggi, A. Fascioli, and D. Ranzato, "Vehicle Detection and Localization in Infra-Red Images," IEEE $5^{\text {th }}$ International Conference on Intelligent Transportation Systems, pp. 141-146, Sep. 3-6, 2002.

[3] I. Pavlidis, V. Morellas, and N. Papanikolopoulos, "A Vehicle Occupant Counting System Based on Near-Infrared Phenomenology and Fuzzy Neural Classification," IEEE Transactions on Intelligent Transportation Systems, Vol. 1, No. 2, pp. 72-85, Jun. 2000.

[4] F. Jahard, D. A. Fish, A. A. Rio, and C. P. Thompson, "Far/Near Infrared Adapted Pyramid-Based Fusion for Automotive Night Vision," IEE $6^{\text {th }}$ International Conference on Image Processing and Its Applications, Vol. 2, pp. 886-890, July 14-17, 1997.

[5] K. Kluge, C. Kreucher and S. Lakshmanan, "Tracking Lane and Pavement Edge Using Deformable Templates," SPIE $12^{\text {th }}$ Annual international Aerosense Symposium, Orlando, Florida, April 1998.

[6] M. Y. Chern and P. C. Hou, "The Lane Recognition and Vehicle Detection at Night for A Camera-Assisted Car on Highway," Proceedings of IEEE International Conference on Robotics and Automation, Vol. 2, pp. 2110-2115, Spet. 14-19, 2003.

[7] R. Taktak, M. Dufaut, and R. Husson, "Vehicle Detection at Night using Image Processing and Pattern Recognition," Proceeds of IEEE International Conference on Image Processing, Vol. 2, pp. 296-300, Nov. 13-16, 1994.

[8] Y. I. Abdel-Aziz, and H. M. Karara, "Direct Linear Transformation from Comparator Coordinates into Object Space Coordinates in Close-Range Photogrammetry", Proceedings of the Symposium on Close-Range Photogrametry, pp. 1-18, Falls Church, VA: American Society of Photogrammetry, 1971.

[9] E. K. Bas and J. D. Crisman, "An Easy to Install Camera Calibration for Traffic Monitoring," IEEE Conference on Intelligent Transportation System, Nov. 9-12, 1997.

[10]J. Canny, "A Computational Approach to Edge Detection," IEEE Transactions on Pattern Analysis and Machine Intelligence, Vol. PAMI8, pp. 679-698, 1986.

[11]Robert M. Haralick and Linda G. Shapiro, Computer and Robot Vision, Vol. 1, Additional-Wesley, 1992.

[12]V. Cantoni, L. Lombardi, M. Porta, and N. Sicard, "Vanishing Point Detection: Representation Analysis and New Approaches, " in Proceedings of 11th IEEE International Conference on Image Analysis and Processing, pp. 90-94, Sept. 26-28, 2001.

[13]S. S. Huang, C. J. Chen, P. Y. Hsiao, and L. C. Fu, "On-Board Vision System for Lane Recognition and Front-Vehicle Detection to Enhance Driver's Awareness," Proceedings of IEEE International Conference on Robotics and Automation, pp. 2456-2461, April 2004. 